\title{
Personalized medicine for radiation therapy
}

\section{"Effectively identifying patients that are sensitive or resistant to radiation therapy could, in the future, be used as a basis for including radiation and tuning the appropriate, therapeutic dose for individual patients in the treatment of cancer."}

\section{KEYWORDS: personalized medicine " predictive biomarkers " radiation therapy - radiosensitivity index = systems biology}

Radiation therapy (RT), either alone or in combination with surgery and/or chemotherapy plays an essential role in treatment and potential curative regimens for most cancers. For example, examination of Surveillance, Epidemiology and End Results (SEER) data (2003-2005) revealed an estimated $29 \%$ of patients with cancer received RT during their initial treatment course [1]. Projections of demand for RT, based on aging and diversification of the US population, is estimated to increase $22 \%$ over the next 10 years [1]. The selection of RT in a treatment plan for a particular patient is currently driven by clinical (e.g., primary site and stage) and/or pathological features (e.g., histology and perineural invasion) defining spectrums of local or regional recurrence risk. Based on these clinical and pathological factors, the omission of RT when indicated leads to significantly worse outcomes at the population level [2]. While RT is one the most effective single modalities in cancer therapy [3], it can have significant side effects both during and after treatment, which can impact both the quality and quantity of life. Although rapid advances in technology, such as intensity modulated RT [4] and proton therapy [5], have led to decreased risk of side effects, these newer modes of RT can be expensive. Thus, there is a significant need for tools to predict the utility of RT in individual patients.

Medical oncology has experienced an explosion of predictive tools for use in guiding therapy decisions. Molecular profiling has become relatively common in supporting clinical decisions regarding chemotherapy. A recent analysis by Frost and Sullivan estimated the value of the US cancer biomarker testing market at US $\$ 7.86$ billion in 2011 [101]. Molecular tests in current clinical use include $K R A S$ and $E G F R$ mutations, which have been shown to be predictive of targeted therapy benefit or nonbenefit in colorectal and lung cancer, respectively [6-9]. More recently, benefit from crizotinib has been shown to occur in a small subpopulation of non-small-cell lung cancer patients that harbor $A L K$ gene rearrangement [10]. It is expected that next-generation sequencing will further impact the number of tests available to support clinical decisions regarding chemotherapy [11,12].

Unlike medical oncology, the personalization of RT can be achieved through several different approaches. The most immediate application is the clinical decision of when RT would be beneficial. Many patients with clinical and pathological indications respond well to RT; however, patients that do not could be considered for other therapies. In addition, low-risk patients may receive additional benefits from therapy that otherwise would not be considered. In addition to a decision-making tool, personalized radiotherapy could also provide alternate dosing schedules for patients that may require more or less total dose of radiation. Effectively identifying patients that are sensitive or resistant to RT could, in the future, be used as a basis for including radiation and tuning the appropriate, therapeutic dose for individual patients in the treatment of cancer.

Given the urgent need to move the field of radiation oncology into the era of personalized medicine, in recent studies we have developed a systems biology model of the intrinsic radiosensitivity of a tumor. We integrated gene expression and cellular radiosensitivity in a database of 48 human cancer cell lines representing a wide array of primary sites, including breast, colon,

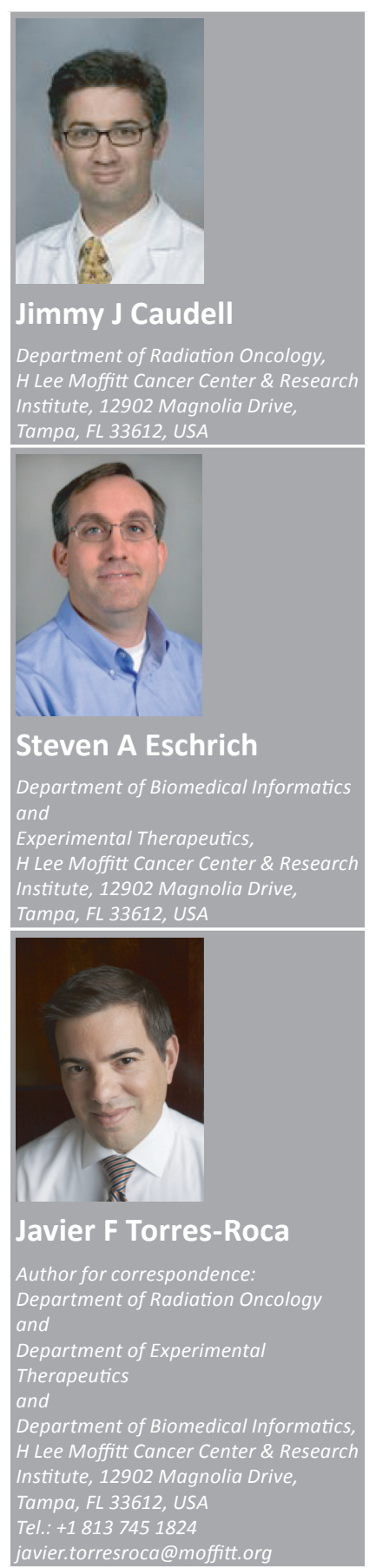

Future : 
leukemia, melanoma, non-small-cell lung, ovarian, renal and prostate cancer to identify 500 genes associated with radiosensitivity $[13,14]$. From these 500 genes, a ten-gene network was identified that we propose plays a central role in determining radiophenotype [14]. We then modeled cellular radiosensitivity as a linear function of gene expression for the ten genes (Box 1). The Radiosensitivity index (RSI) is modeled on the cellular survival after RT in the cell lines, therefore a low RSI indicates a higher response to RT (i.e., radiosensitive).

\section{"The radiosensitivity index, or any other assay that can predict response to radiotherapy, will have a transformative impact on the practice of radiation oncology."}

The ten-gene RSI model appears to represent intrinsic radiation sensitivity within in vitro models of cancer. Importantly, the model has also been validated from patient tumors in four different primary sites in five different cohorts. First, in 14 patients with rectal cancer treated with radiation and chemotherapy followed by surgery, pretreatment tumor prediction of RSI was associated with response to radiochemotherapy. Response was defined as a decrease of one $\mathrm{T}$ stage in the primary tumor between pretreatment endorectal ultrasound and pathological evaluation. Responders to preoperative radiochemotherapy were more radiosensitive (lower RSI) as deemed by RSI (responders vs nonresponders: RSI: 0.32 vs $0.46 ; \mathrm{p}=0.03$ ) [15]. Second, a similar result was observed in pretreatment specimens from 12 patients with esophageal cancer treated with radiation and chemotherapy, with or without surgery, (responders vs nonresponders: RSI, 0.37 vs $0.50 ; \mathrm{p}=0.05$ ) [15]. Third, in a cohort of 92 patients with locally advanced head and neck cancer patients treated with radiochemotherapy, RSI was associated with 2-year locoregional control $-86 \%$ in radiosensitive patients compared with $61 \%$ in radioresistant patients $(\mathrm{p}=0.05)$. Importantly, a number of other potential classifiers were previously tested in the same cohort, including wound, hypoxia, chromosomal instability, and stem-cell-based signatures, and were not associated with prognosis [16].

Finally, RSI was tested in two different cohorts of breast cancer patients [17]. The first cohort of
159 patients was heterogeneously treated: surgery, with or without radiotherapy, with or without chemotherapy or hormonal therapy. Breast cancer patients treated with surgery and radiotherapy who were determined to be RSI radiosensitive had an improved 5-year relapse-free survival rate (95 vs $75 \% ; \mathrm{p}=0.02$ ). In the same cohort, RSI was not predictive of outcomes in patients treated without radiotherapy. A second cohort of 344 lymph-node negative breast cancer patients treated with surgery with or without radiotherapy was also examined. RSI radiosensitive patients had an improved distant metastasis-free survival rate compared with radioresistant patients (77 vs $64 \%$ at 5 years; $p=0.04$ ). Again, RSI was not predictive in breast cancer patients treated with surgery alone.

We have demonstrated that a biomarker (RSI) derived in cell lines to predict radiation sensitivity is associated with improved outcomes for patients treated with radiotherapy. This is seen for a number of different end points (response rate, locoregional control, distant metastasis-free survival and relapse-free survival) and different tumor types (rectal, esophagus, head and neck and breast). Thus, it is reasonable to hypothesize that RSI is a tissue-independent predictor of radiosensitivity. The RSI assay is currently undergoing further clinical utility validation in a number of different cancers with an eye towards US FDA approval for clinical use.

\section{"Future clinical trials may seek to modulate the dose of radiotherapy based on radiosensitivity; a lower dose of radiotherapy should result in less risk of short-and long-term side effects."}

The RSI has been developed to predict RT therapeutic benefit independent of the disease site. At the same time, the clinical application and benefit of RT varies widely across disease sites. To that end, clinical development must be addressed in a disease-specific manner. One clinical example where RSI can have a significant impact is rectal cancer. The current standard of care for patients with stage 2 or 3 rectal cancer is to undergo preoperative concurrent chemo-RT followed by surgery $[18,19]$. However, approximately $40-50 \%$ of the individuals that receive preoperative chemo-RT, experience no response to treatment and, thus, 
undergo preoperative therapy for no benefit. We think that RSI can accurately identify these patients and therapeutic decisions can be adjusted accordingly (i.e., avoiding RT and proceeding directly to surgery).

The RSI, or any other assay that can predict response to radiotherapy, will have a transformative impact on the practice of radiation oncology. We envision RSI may impact clinical decisions in radiation oncology in multiple ways - for example, in patients who are in the low RSI spectrum (radiosensitive) RT is predicted to be effective. Depending on the disease site it may be reasonable for these patients to undergo radiotherapy rather than surgery. In contrast, patients in the high end of the RSI spectrum (radioresistant) may be better off treated without radiotherapy. A clinical example would be in rectal or esophageal cancer where radioresistant patients may proceed directly to surgery without preoperative RT. Finally, we think there might be patients that will fall in the intermediate range for RSI and these are the patients where RT dose modifications or concurrent chemotherapy might significantly impact their clinical outcome. Future clinical trials may seek to modulate the dose of radiotherapy based on radiosensitivity; a lower dose of radiotherapy should result in less risk of short- and long-term side effects. In addition, future clinical trials may be directed at methods to overcome radioresistance, for example, integration of surgery with or without radiotherapy, increasing the dose of radiotherapy, or use of concurrent chemotherapy or molecularly targeted therapies aimed at overcoming radioresistance. RSI, which is based on a ten-gene network culled from 500 genes associated with radiosensitivity, offers an abundance of initial hypotheses and targets from which to begin.

In summary, radiotherapy is, and will continue to be, an integral part of both curative and palliative treatments for cancer. It is imperative that the field of radiation oncology enter the era of personalized medicine such that clinicians and patients undergo more tailored, less toxic and more cost-effective care.

\section{Financial \& competing interests disclosure}

Funding was provided by the NIH (R21CA101355/ R21CA135620, K08CA108926), US Army Medical Research and Materiel Command, National Functional Genomics Center (170220051) and the Bankhead-Coley Foundation (09BB-22). JF Torres-Roca and SA Eschrich hold an awarded patent and two pending patents on the technology described in this editorial. In addition, they are co-founders and officers of Cvergenx, Inc., a company that holds the exclusive commercial rights to the assay described. The authors have no other relevant affiliations or financial involvement with any organization or entity with a financial interest in or financial conflict with the subject matter or materials discussed in the manuscript apart from those disclosed.

No writing assistance was utilized in the production of this manuscript.

\section{References}

1 Smith BD, Haffty BG, Wilson LD, Smith GL, Patel AN, Buchholz TA. The future of radiation oncology in the United States from 2010 to 2020 : will supply keep pace with demand? J. Clin. Oncol. 28(35), 5160-5165 (2010).

2 Hamidi M, Moody JS, Kozak KR. Refusal of radiation therapy and its associated impact on survival. Am. J. Clin. Oncol. 33(6), 629-632 (2010).

3 Tepper JE, Sartor CI. Radiation therapy and biologics: a ripe opportunity? Nat. Clin. Pract. Oncol. 3(9), 463 (2006).

4 Nutting CM, Morden JP, Harrington KJ et al. Parotid-sparing intensity modulated versus conventional radiotherapy in head and neck cancer (PARSPORT): a Phase 3 multicentre randomised controlled trial. Lancet Oncol. 12(2), 127-136 (2011).

5 Zietman AL, DeSilvio ML, Slater JD et al. Comparison of conventional-dose vs highdose conformal radiation therapy in clinically localized adenocarcinoma of the prostate a randomized controlled trial. JAMA 294, 1233-1239 (2005).

6 Amado RG, Wolf M, Peeters $M$ et al. Wild-type KRAS is required for panitumumab efficacy in patients with metastatic colorectal cancer. J. Clin. Oncol. 26(10), 1626-1634 (2008).

7 Karapetis CS, Khambata-Ford S, Jonker DJ et al. K-ras mutations and benefit from cetuximab in advanced colorectal cancer. N. Engl. J. Med. 359(17), 1757-1765 (2008).

8 Mitsudomi T, Morita S, Yatabe Y et al. Gefitinib versus cisplatin plus docetaxel in patients with non-small-cell lung cancer harbouring mutations of the epidermal growth factor receptor (WJTOG3405): an open label, randomised Phase 3 trial. Lancet Oncol. 11(2), 121-128 (2010).

9 Maemondo M, Inoue A, Kobayashi K et al. Gefitinib or chemotherapy for non-smallcell lung cancer with mutated EGFR. N. Engl. J. Med. 362(25), 2380-2388 (2010).
10 Shaw AT, Yeap BY, Solomon BJ et al. Effect of crizotinib on overall survival in patients with advanced non-small-cell lung cancer harbouring $A L K$ gene rearrangement: a retrospective analysis. Lancet Oncol. 12(11), 1004-1012 (2011).

11 Cheng L, Lu W, Kulkarni B et al. Analysis of chemotherapy response programs in ovarian cancers by the next-generation sequencing technologies. Gynecol. Oncol. 117(2), 159-169 (2010).

12 Sastre L. New DNA sequencing technologies open a promising era for cancer research and treatment. Clin. Transl Oncol. 13(5), 301-306 (2011).

13 Torres-Roca JF, Eschrich S, Zhao H et al. Prediction of radiation sensitivity using a gene expression classifier. Cancer Res. 65(16), 7169-7176 (2005).

14 Eschrich S, Zhang H, Zhao H et al. Systems biology modeling of the radiation sensitivity network: a biomarker discovery platform. Int. J. Radiat. Oncol. Biol. Phys. 75(2), 497-505 (2009). 
15 Eschrich SA, Pramana J, Zhang $\mathrm{H}$ et al. A gene expression model of intrinsic tumor radiosensitivity: prediction of response and prognosis after chemoradiation. Int. J. Radiat. Oncol. Biol. Phys. 75(2), 489-496 (2009).

16 Pramana J, Van den Brekel MW, van Velthuysen ML et al. Gene expression profiling to predict outcome after chemoradiation in head and neck cancer. Int. J. Radiat. Oncol. Biol. Phys. 69(5), 1544-1552 (2007).
17 Eschrich SA, Fulp WJ, Pawitan Y et al. Validation of a radiosensitivity molecular signature in breast cancer. Clin. Cancer Res. 18(18), 5134-5143 (2012).

18 Sauer R, Becker H, Hohenberger W et al. Preoperative versus postoperative chemoradiotherapy for rectal cancer. $N$. Engl. J. Med. 351, 1731-1740 (2004).

19 Sebag-Montefiore D, Stephens RJ, Steele R et al. Preoperative radiotherapy versus selective postoperative chemoradiotherapy in patients with rectal cancer (MRC CR07 and
NCIC-CTG C016): a multicentre, randomised trial. Lancet 373(9666), 811-820 (2009).

\section{Website}

101 Frost \& Sullivan. Analysis of the US Cancer Biomarker Testing Market. Targeted Sequencing of Tumor Mutations is on the Horizon.

www.frost.com/c/10024/sublib/displayreport.do?id=NB1D-01-00-00-00 